\title{
Treatment of oligodendroglioma: An update
}

\author{
Nina A. Paleologos ${ }^{1}$ and J. Gregory Cairncross \\ Evanston Northwestern Healthcare, Evanston, IL 60201 [N.A.P.]; London Regional Cancer Centre, London, \\ Ontario, Canada N6A 4 L6 [J.G.C.]
}

Oligodendrogliomas are rare primary brain tumors. Significant attention has recently been focused on these interesting neoplasms because of their unique chemosensitivity and the durability of some of these responses. Surgery and radiation continue to play important roles in the treatment of oliogodendrogliomas. Molecular genetic analyses have given new insight into the allelic deletions that distinguish these tumors and their progression from indolent to more aggressive forms. In the future, molecular genetic analysis may guide therapeutic decisions concerning patients with oligodendroglioma and may help us learn more about how to best treat other malignant brain neoplasms. Neuro-Oncology 1, 61-68, 1999 (Posted to Neuro-Oncology [serial online], Doc. 98-10, January 19, 1999. URL <neuro-oncology.mc. duke.edu>)

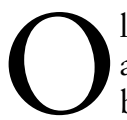

ligodendrogliomas are much less prevalent than astrocytomas, representing $4-5 \%$ of primary brain tumors (Mørk et al., 1985) and 4-15\% of primary glial tumors (Kallio, 1990; Soffietti et al., 1994). They have been appreciated as a distinct neoplastic entity since the 1920s (Bailey and Bucy, 1929; Bailey and Cushing, 1926; Bailey and Hiller, 1924). While they have always been intriguing, because of their unique neuropathologic and clinical features, they were not treated differently than astrocytomas until the past decade. Significant attention has now been focused on oligodendrogliomas because of their unique chemosensitivity (Cairncross and Macdonald, 1988) and the link between drug response and allelic loss of $1 \mathrm{p}$, the latter recognized only recently (Cairncross et al., 1998).

Received 31 July 1998, accepted 1 October 1998.

${ }^{1}$ Address correspondence and reprint requests to Nina A. Paleologos, MD, Division of Neurology, Evanston Hosptial, 2650 Ridge Avenue, Evanston, IL 60201.

\footnotetext{
${ }^{2}$ Abbreviations used are as follows: CCNU, 1-(2-chloroethyl)-3cyclohexyl-1-nitrosourea; FLAIR, fluid attenuated inversion recovery; $\mathrm{PCV}$, procarbazine/CCNU [lomustine]/vincristine; RT, radiation therapy; TTP, time to tumor progression.
}

Oligodendrogliomas typically have an indolent course, yet the majority are incurable tumors from which most patients will ultimately die. Median survival may be greater than 10 years in low-grade oligodendrogliomas (Leighton et al., 1997), whereas patients with anaplastic oligodendrogliomas have a median survival time after surgery of less than 5 years (Celli et al., 1994; Gannett et al., 1994; Ludwig et al., 1986; Nijjar et al., 1993; Shaw et al., 1992, 1994; Smith et al., 1983; Wilkinson et al., 1987).

The median age at diagnosis is 40-50 years, with onequarter of patients being diagnosed after the age of 60 and very few before the age of 18 (Bullard et al., 1987; Celli et al., 1994; Ludwig et al., 1986; Mørk et al., 1985; Shaw et al., 1992). There is a slight predominance of occurrence of oligodendrogliomas in male patients. Occasional familial clustering is seen (Kros et al., 1994a; Roelvink et al., 1986; Roosen et al., 1984); however, most occur sporadically without any clear pattern of inheritance. If there are genetic risk factors for the development of oligodendrogliomas, they are not yet obvious.

The majority of oligodendrogliomas and mixed oligoastrocytomas are single lesions located in the cerebral hemispheres and typically involve the subcortical white matter and overlying cortex (Celli et al., 1994; Ludwig et al., 1986; Mørk et al., 1985; Shaw et al., 1992; Sun et al., 1988). While oligodendrogliomas may eventually invade deep grey structures, only rarely are they confined to these areas (Russell and Rubenstein, 1989). Occasionally, they occur in the cerebellum, brainstem, spinal cord, or optic nerve; however, these locations are most unusual (Fortuna et al., 1980; Garza-Mercado et al., 1987; Nijjar et al., 1993; Pagni et al., 1991). Intraventricular oligodendrogliomas have been reported (Dolinskas and Simeone, 1987; Lee and Kelly, 1990). However, many central neurocytomas were once mistaken for intraventricular oligodendrogliomas, the former distinguished by the presence of Homer-Wright rosettes and synaptophysin expression (Russell and Rubenstein, 1989; Yuen et al., 1992). At times, extension of an intraparenchymal oligodendroglioma into the leptomeninges is seen. Considering their extensive cortical involvement, this is not surprising. Recently there were two reports of oligodendroglioma confined solely to the 
leptomeninges without parenchymal brain or spinal cord involvement (Chen et al., 1995; Rogers et al., 1995). Systemic metastases are rarely seen, occurring usually in patients with anaplastic tumors who have enjoyed longer survival and who have been treated with intensive multimodality therapy (Macdonald et al., 1989). Dissemination at time of relapse, within and outside the nervous system, may become more common in the future as local therapies improve and patients live longer.

As with all brain tumors, the symptoms and signs are related to tumor location and size and can be focal or due to raised intracranial pressure. Seizures occur very commonly as a presenting symptom in more than $50 \%$ of patients and will occur at some time during the course of the illness in up to $88 \%$ of patients (Ludwig et al., 1986; Mørk et al., 1985; Nijjar et al., 1993; Shaw et al., 1992). While seizures occur in all supratentorial brain tumors, their particularly high incidence in patients with oligodendrogliomas is thought to be due to the propensity of these tumors to involve the cerebral cortex. The duration of time between onset of symptoms and diagnosis varies widely, from hours to years (Mørk et al., 1985; Nijjar et al., 1993; Shaw et al., 1992), with delayed diagnosis becoming increasingly uncommon as radiologic imaging studies have improved and their availability has increased (Celli et al., 1994; Lee and Tassel, 1989).

\section{Neuroimaging}

Neuroimaging studies are never specific but frequently can suggest oligodendroglioma. Older studies found that plain skull radiographs revealed calcification in about one-half of the cases. CT typically demonstrates a welldemarcated hypodense mass, often near the cortical surface. Frequently, intratumoral calcification is seen and occasionally subclinical or symptomatic hemorrhage is detected (Lee and Tassel, 1989; Vonokakos et al., 1979). Contrast enhancement, when seen, is typical of aggressive or anaplastic tumors. MRI does not detect intratumoral calcification as effectively as CT, but in all other respects is the neuroimaging modality of choice. The T1 images usually reveal a hypointense mass, and the T2 images disclose a hyperintense lesion that extends beyond what is appreciated on most CT scans (Margain et al., 1991). FLAIR ${ }^{2}$ imaging may prove to be even more effective in showing extent of disease. FLAIR images usually reveal a hyperintense mass that is larger than is seen on T2 images. As with CT, anaplastic or aggressive oligodendrogliomas usually enhance following intravenous gadolirium infusion (Fig. 1). Most enhancing oligodendrogliomas do so in a patchy or homogeneous fashion; ring-enhancing tumors are uncommon and seldom respond to treatment (Cairncross et al., 1998).

\section{Histology}

As discussed by Bigner et al. (1999) elsewhere in this volume, the histologic appearance of oligodendrogliomas is distinctive; moreover, in our experience, this unique morphology, when textbook in nature, is highly predictive of response to chemotherapy. On the other hand, oligodendrogliomas with atypical morphology, mixed histology, or a resemblance to small-cell glioblastomas are much less likely to be chemosensitive. Accurate histopathologic diagnosis is important. There is still some controversy regarding histologic criteria for the diagnosis and which grading system is best. Molecular diagnosis, particularly if predictive of response or survival, will become an important adjunct to traditional histopathologic diagnosis (Cairncross et al., 1998).

\section{Treatment}

The treatment of oligodendroglioma is evolving and should depend on the circumstances of an individual patient. Decisions are usually based on tumor location and size, patient symptoms, extent of surgical resection possible, degree of histologic anaplasia, and tumor aggressiveness. In the future, molecular genetic analyses may influence treatment selection. Currently, there is a trend for less intense treatment for patients with tumors behaving indolently and more intense therapy for patients whose tumors are anaplastic, recurrent, or aggressive. The principal therapies for oligodendrogliomas are surgery, radiotherapy, and chemotherapy. Increasingly, chemotherapy is replacing radiation as the initial postoperative treatment because of a better CNS toxicity profile. Radiation may be the more potent tumor therapy, but it has significant brain toxicity in some cases.

\section{Surgery}

Historically, surgical resection has been the mainstay of treatment for oligodendroglioma and establishes the histopathologic diagnosis. Resection has been found, in several retrospective studies, to be a significant independent prognostic factor for survival in patients with pure oligodendroglioma and mixed oligoastrocytoma (Celli et al., 1994; Lindegaard et al., 1987; Mørk et al., 1985; Shaw et al., 1992, 1994). In one retrospective study, patients who underwent gross total resection had a median survival time and 5- and 10-year survival rates of 12.6 years and 74 and $59 \%$, respectively, compared with 4.9 years and 46 and $23 \%$, respectively, for patients who had subtotal resection (Shaw et al., 1992). In another retrospective study of 105 evaluable patients, only the subset with benign histology, seizures as initial symptoms, and normal neurologic examination at diagnosis lived significantly longer after total tumor resection compared with patients having subtotal resection or biopsy (Celli et al., 1994). For the entire group, Celli et al. (1994) did not find extent of resection to be a positive prognostic factor, but did observe that surgery done in the era of modern neuro-imaging was a positive prognostic factor.

Today, it is generally thought that the most extensive surgical resection compatible with preserved neurologic 

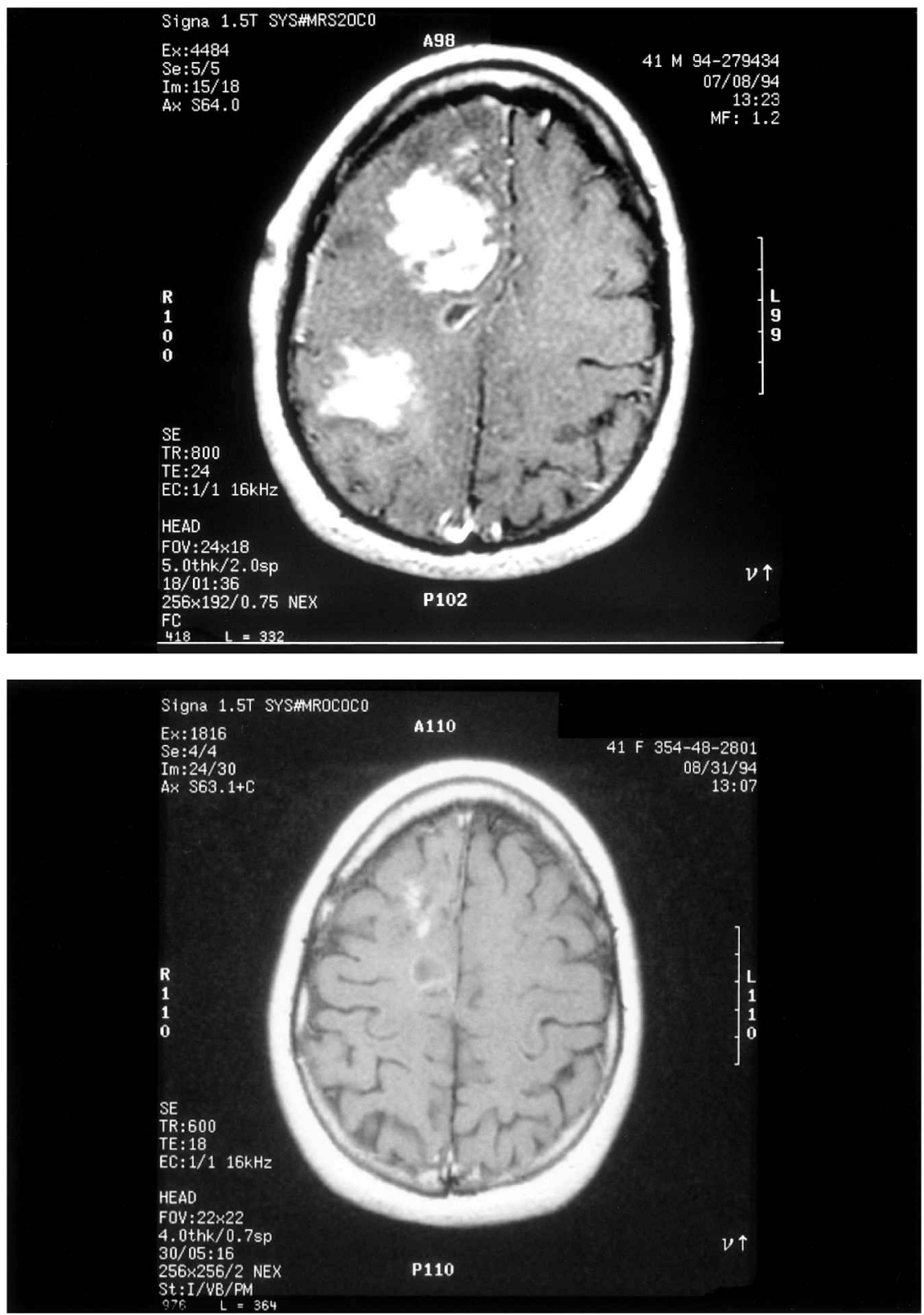

Fig. 1: Anaplastic oligodendroglioma before and after one cycle of intensive procarbazine/CCNU [lomustine]/vincristine.

function should be performed. The level of risk to neurologic function that is considered acceptable depends on many factors. An important consideration is whether the tumor is behaving indolently or aggressively; admittedly, a distinction that can be difficult to make. Cortical mapping in awake patients may facilitate more complete and safer surgical resection in patients with tumors in or near eloquent areas of brain. In patients with low-grade tumors located in non-eloquent areas, gross total resection alone may be the optimal initial therapy; however, close monitoring of these patients with serial scans is advised to detect early on tumors that are behaving more aggressively than anticipated, based on their initial clinical, radiologic, and pathologic features. Some patients may then benefit from reoperation (again depending on location of the tumor and technical feasibility of resection with low risk to neurologic function) followed by radiotherapy or chemotherapy or both.

\section{Radiation Therapy}

The role of radiation therapy in the treatment of oligodendrogliomas is not entirely clear. No prospective randomized trials have been performed to assess the efficacy of radiation therapy. Nevertheless, radiation therapy is almost certainly effective, although there may be subsets of patients for whom it may be more prudent to defer treatment. In 1992, Shaw et al. reviewed the records of 81 patients and found no overall survival benefit in 63 patients with oligodendrogliomas who received postop- 
erative radiation. However, in the subset of patients who had a subtotal resection, postoperative radiation appeared to improve survival. Also noted was a doseresponse relationship to radiation with patients who received in excess of $50 \mathrm{~Gy}$, faring better than those who received lower doses. In an earlier study by Lindegaard et al. (1987), a dose-response effect was not seen, although the median survival and 5-year survival were better in patients receiving radiation (38 months, 36\%) compared with those having surgery alone (26.5 months, $27 \%$ ). The 8 -year survival rates were similar, however, $17 \%$ of patients receiving radiation compared with $14 \%$ receiving surgery alone, suggesting that there was little effect on long-term survival. Additionally, in the same study, radiation therapy did not seem to improve prognosis for patients in whom the surgeon considered the tumor to have been totally resected. Mørk et al. (1985) also showed the benefit of radiation therapy, but also did not show a benefit in patients with gross total resection. Most evidence from other retrospective studies also supports the use of postoperative radiation, showing improved median survival times and prolonged 5- and 10-year survival rates, although not in all groups (Gannett et al., 1994; Mørk et al., 1985; Shimizu et al., 1993; Wallner et al., 1988). Several studies do not reflect these findings and show no beneficial effect of radiation therapy (Bullard et al., 1987; Kros et al., 1994b; Nijjar et al. 1993; Reedy et al. 1983).

Evaluating these studies to determine treatment decisions is difficult because of their retrospective uncontrolled nature, small sample sizes, heterogeneous patient populations, broad range of radiation dosages, and wide range of years predating the modern neuroimaging era, among other factors. Contributing to the role of radiation controversy is the unclear definition of tumor pathology; nor is grade or degree of anaplasia always clear or comparable between groups of patients within the population reviewed as a whole or from study to study.

In low-grade oligodendroglioma, as in low-grade astrocytoma, the controversy seems more crucial. These patients frequently have normal or near normal function, tumors with indolent natural courses, and tumors that involve large areas of brain requiring large treatment ports, perhaps exposing more normal brain to the deleterious long-term effects of radiation. In an interesting recent study, Celli et al. (1994) looked at survival data related to the clinical syndrome rather than the grade of tumor or the extent of resection, and observed that postoperative radiation prolonged median survival times and 5-year survival rates for many patients with oligodendrogliomas, but not for the subset of patients with indolent tumors. They did not define these patients by grade or degree of anaplasia, but rather by behavior, these patients having had seizures only and a normal neurologic status. They considered separately low-grade oligodendroglioma patients who had symptoms other than seizures or who had intracranial hypertension.

Until a well-organized prospective randomized trial is done, careful review of retrospective data and an understanding of the limitations of these studies are needed to treat patients optimally. The authors cur- rently recommend postoperative radiation therapy for patients with anaplastic tumors and enlarging tumors, but increasingly recommend neoadjuvant PCV chemotherapy for these patients. In patients with low-grade neoplasms that have been completely or largely resected, or who have relatively asymptomatic tumors that are not growing, it seems prudent to defer both chemotherapy and radiation, provided they are followed closely with serial neuro-imaging studies. It is unclear whether it is safe to defer radiation in patients with anaplastic tumors that have had gross total resection and other treatment such as chemotherapy.

\section{Chemotherapy}

Through the 1970s and 1980s, phase II and phase III chemotherapy trials for glioma included both astrocytomas and oligodendrogliomas. Many did not actively include oligodendrogliomas, nor were oligodendrogliomas specifically excluded. Because of their relatively small numbers oligodendrogliomas were, for the most part, lost in the crowd. It was not until relatively recently that their unique and predictable response to chemotherapy was first appreciated. In 1988, Cairncross and Macdonald reported that recurrent anaplastic oligodendroglioma responded predictably to nitrosourea-based chemotherapy, especially PCV, a regimen developed by Levin et al. (1980). They reported eight consecutive patients with recurrent malignant oligodendroglioma who were treated with chemotherapy; all of them responded to treatment (one completely and seven partially). All responses were durable, lasting $>30$ to $>78$ weeks. Six of the eight patients were treated with PCV, one with carmustine (BCNU) and one with diaziquone (AZQ). Subsequently, Saarinen et al. (1990) reported a complete response to thiotepa in a pediatric patient with a malignant oligodendroglioma that had recurred for a third time. Also in 1990, The Central Nervous System Cancer Consortium conducted a phase II study of intravenous melphalan for treatment of recurrent anaplastic glioma and found that patients with anaplastic oligodendrogliomas were more likely to respond $(55 \%)$ than were those with nonoligodendrogliomas (11\%) (Brown et al., 1990).

Encouraged by successful chemotherapy for recurrent disease (Fig. 1), Macdonald et al. (1990) began treating patients who had newly diagnosed aggressive oligodendrogliomas by using chemotherapy before radiotherapy and again observed responses. In this small series, the term "aggressive" was used to describe both anaplastic oligodendrogliomas and nonanaplastic oligodendrogliomas that were symptomatic, enlarging, and contrast-enhancing.

Anaplastic mixed oligodendrogliomas also appear to be chemosensitive tumors. Glass et al. (1992) reported complete or partial responses to PCV chemotherapy in 9 of 10 patients with mixed oligoastrocytoma treated before irradiation, and 2 of 4 treated after irradiation (no recurrence). Other reports appear to confirm this finding (Kyritsis et al., 1993), but uncertainty regarding the chemosensitivity of mixed gliomas has been 
Table 1. Standard and intensive PCV chemotherapy

\begin{tabular}{ll} 
Regimen & \multicolumn{1}{c}{ Dose and schedule } \\
\hline Standard PCV & $110 \mathrm{mg} / \mathrm{m}^{2}$, p.o., day 1 \\
CCNU & $60 \mathrm{mg} \cdot \mathrm{m}^{-2} \cdot$ day ${ }^{-1}$, p.o., days $8-21$ \\
Procarbazine & $1.4 \mathrm{mg} / \mathrm{m}^{2}$ (maximum, $2.0 \mathrm{mg}$ ), i.v., days 8 and 29 \\
Vincristine & \\
Intensive PCV & $130 \mathrm{mg} / \mathrm{m}^{2}$, p.o., day 1 \\
CCNU & $75 \mathrm{mg} \cdot \mathrm{m}^{-2} \cdot$ day ${ }^{-1}$, p.o., days $8-21$ \\
Procarbazine & $1.4 \mathrm{mg} / \mathrm{m}^{2}$ (no maximum), i.v., days 8 and 29 \\
Vincristine & Every 6 weeks \\
\hline
\end{tabular}

Abbreviations: CCNU, 1-(2-chloroethyl)-3-cyclohexyl-1-nitrosourea; PCV, procarbazine/CCNU [lomustine]/vincristine.

expressed by others. The lack of consistency in the diagnosis of mixed tumors leaves open the possibility that responding oligodendrogliomas at one center might be called "mixed tumors" at another. Likewise, nonresponding mixed tumors at one center might be called "astrocytomas" at another. Molecular analysis may yet clarify these issues. Genetically speaking, mixed gliomas are of two types, those with $1 \mathrm{p}$ deletions and those with p53 mutations (Maintz et al., 1997); the former are likely sensitive to PCV (Cairncross et al., 1998).

Reports on small numbers of patients from single institutions may overestimate the effectiveness of new or newly appreciated treatments. Consequently, the National Cancer Institute of Canada conducted a multicenter phase II trial of intensive PCV for treatment of new or recurrent pure anaplastic oligodendrogliomas and observed a $75 \%$ response rate $(38 \%$ complete $)$ (Cairncross et al., 1994). At the time the study was reported, the median time to tumor progression (TTP) had not been reached for patients with complete response and was at least 25.2 months. TTP for partial responders (38\% of patients) was 14.2 months. An update on that study 1 year later reported the TTP for complete responders to be at least 36.1 months (Cairncross and Eisenhauer, 1995). It is unknown whether the intensive formulation of PCV is superior to the standard formulation (Table 1). Both formulations can be given safely, although intensive PCV is certainly more toxic (Cairncross et al., 1994; Mason et al., 1996).

Increasingly, newly diagnosed patients with anaplastic and aggressive oligodendroglioma (as defined by Macdonald et al., 1990) are being given PCV before radiation therapy. In a retrospective analysis of newly diagnosed patients treated with neoadjuvant (pre-radiotherapy) PCV or intensive PCV, $75 \%$ of patients responded. Twenty-five percent needed early RT due to chemotherapy resistance or toxicity. Neoadjuvant chemotherapy, deferring RT while prescribing four to six cycles of PCV, seemed safe for many patients, especially those who were complete responders or who had gross total resections (Paleologos et al., 1995). Whether RT can be deferred indefinitely for those subsets remains unclear. Deferring RT in those patients with gross total resection is also supported by studies that did not show a clear benefit of early postoperative RT in that group of patients (Lindegaard et al., 1987; Mørk et al., 1985; Shaw et al., 1992).
To determine the value of adjuvant chemotherapy in addition to radiation, the Radiation Therapy Oncology Group is conducting a phase III randomized trial comparing PCV plus radiation versus radiation alone for patients with newly diagnosed pure and mixed anaplastic oligodendrogliomas. This trial has accrued in excess of 160 patients (accrual goal, 292).

Little is known about what agents may be best used as salvage therapy for tumor progression in patients previously treated with PCV or other chemotherapy. Peterson et al. (1996), reporting for the Oligodendroglioma Tumor Study Group, observed responses to etoposide plus cisplatin in $40 \%$ of patients who were initially treated with PCV and then relapsed. In that report, seven of eight patients who had been exposed to regimens other than PCV responded to PCV at the time of progression. In a few cases only, re-treating with PCV after prior administration of PCV seemed ineffective. Soffietti et al. (1995) treated with carboplatinum seven patients who had progressed after initial PCV chemotherapy. One of those patients had a partial response with clinical improvement lasting more than 6 months.

While most newly diagnosed patients respond to PCV, with some responses being durable, most patients are not cured. Because of this and because treatment is limited by bone marrow reserves, a strategy for improving treatment might include high-dose chemotherapy with autologous bone marrow or peripheral stem cell reconstitution. Individual patients with complete responses to this approach have been reported (Kedar et al., 1994; Saarinen et al., 1990). To this end, The Oligodendroglioma Tumor Study Group has initiated phase II multicentered trials of high-dose chemotherapy followed by hematopoietic reconstitution for patients with recurrent and newly diagnosed pure or mixed anaplastic or aggressive oligodendroglioma. Patients with recurrent tumors occasionally have durable responses, but may experience significant toxicity, including profound anorexia and chronic encephalopathy. Preliminary experience with newly diagnosed patients in whom radiation is deferred includes tolerance of high-dose thiotepa and some long disease-free survival times with good neurologic function. The wasting/encephalopathy syndrome, observed in some patients with recurrent anaplastic oligodendroglioma treated this way, has not been seen (Cairncross et al., 1997). 
In addition to dose intensification protocols for anaplastic and aggressive tumors, the use of PCV to treat patients with lower grade, more indolent tumors is beginning to be explored. Experience is limited however. In the series reported by Glass et al. (1992), two patients with oligodendroglioma were treated with PCV, one before and one after irradiation. The one treated before radiotherapy had stable disease after three cycles and the other, treated after radiotherapy and one cycle of melphalan, had rapidly progressive disease and died 8 months after PCV initiation. Postmortem examination revealed that a small portion of the tumor was a grade IV astrocytoma. Whether either of these cases had contrast enhancement on neuro-imaging studies is unclear. Paleologos et al. (1994) reported on five patients with low-grade oligodendroglioma, all with nonenhancing masses on MRI, all with grade I (one patient) or grade II (four patients) oligodendroglioma confirmed by two independent neuropathologists. Three of the five had partial responses. One of these had a significant decrease in seizure frequency, which was ascribed to the PCV. The other two had stable disease. Mason et al. (1996) treated nine patients. One patient was treated at time of recurrence after radiotherapy and had what the authors referred to as "prominent enhancement" on MRI. Eight patients were treated at presentation or shortly thereafter. Two of those patients had enhancement on MRI; the other six had histologically confirmed low-grade oligodendroglioma and no enhancement on MRI. Four of the eight patients treated at time of presentation responded to PCV. Only one of those is described as having significant enhancement. Others have also noted responses in low-grade oligodendrogliomas treated with PCV (Soffietti et al., 1994). We have seen that the low-grade tumors respond less dramatically and more slowly (over multiple cycles) than do anaplastic or aggressive oligodendrogliomas.

While much has been done in the past decade, there are many questions still unanswered regarding the best treatment of patients with oligodendroglioma. Cur- rently, PCV plays an important role, but we still do not know whether there are better or less toxic regimens. The long-term effects of PCV on patients is unknown. Concern regarding the possibility of secondary malignancies or other toxicity in the long-term survivor is particularly warranted when considering treatment of patients with low-grade oligodendroglioma who have inherently longer survival times. Reduced bone marrow reserves in these patients may also limit later treatment options. A randomized prospective trial comparing chemotherapy with radiation as initial treatment in symptomatic patients would be needed to determine which method is superior and associated with less toxicity. Studies that address these issues may be possible in the future using intergroup trial mechanisms.

The appreciation that oligodendrogliomas respond to chemotherapy, particularly PCV, has had a significant impact on patient care and has been one of the more interesting recent developments in neuro-oncology. The recent discovery of a specific molecular diagnostic marker associated with chemosensitivity will be another important development in oligodendroglioma therapeutics. The reason for their unique chemosensitivity is unknown. Because oligodendrogliomas appear less permeable than astrocytomas, which are relatively chemoresistant, better drug delivery does not appear to be the explanation (Paleologos et al., 1992). Evidence suggests that oligodendroglia may have impaired DNA repair mechanisms, rendering them vulnerable to drugs that alkylate DNA. Low levels of $\mathrm{O}^{6}$-alkylguanine-DNA alkyltransferase, a DNA repair enzyme, have been found in oligodendroglial lineage cells in the rat (Nutt et al., 1995) and some human oligodendroglial tumors (Frosina et al., 1990; Nutt et al., 1995; Wiestler et al., 1984). These speculations notwithstanding, our understanding of the biochemical basis of oligodendroglioma chemosensitivity will likely await the cloning of genes on $1 \mathrm{p}$ and $19 \mathrm{q}$, which give rise to this fascinating clinical, morphologic, and treatment-sensitive glioma.

\section{References}

Bailey, P., and Bucy, P.C. (1929) Oligodendrogliomas of the brain. J. Pathol. 32,735-751.

Bailey, P., and Cushing, H. (1926) Tumors of the Glioma Group. Philadelphia: Lippincott.

Bailey, P., and Hiller, G. (1924) The interstitial tissues of central nervous system: A review. J. Nerv. Ment. Dis. 337-361.

Brown, M.T., Cairncross, J.G., Vick, N.A., Mcdonald, D.R., Friedman, H.S., Dropcho, E.J., Schold, S.C., Jr.(1990) Differential response of recurrent oligodendrogliomas vs astrocytomas to intravenous (iv) melphalan. Neurology 40 (Suppl. 1), 397-398. (Abstract)

Bullard, D.E., Rawlings, C.E., III, Phillips, B., Cox, E.B., Schold, S.C., Jr., Burger, P., and Halperin, E.C. (1987) Oligodendroglioma: An analysis of the value of radiation therapy. Cancer 60, 2179-2188.

Cairncross, J.G., and Eisenhauer, E.A. (1995) Response and control: Lessons from oligodendroglioma.J. Clin. Oncol. 13, 2475-2476. (Letter)

Cairncross, J.G., and Macdonald, D.R. (1988) Successful chemotherapy for recurrent malignant oligodendroglioma. Ann. Neurol. 23, 360-364.

Cairncross, G., MacDonald, D., Ludwin, S., Lee, D., Cascino, T., Buckner, J., Fulton, D., Dropcho, E., Stewart, D., and Schold, C., Jr. (1994) Chemo- therapy for anaplastic oligodendroglioma. National Cancer Institute of Canada Clinical Trials Group. J. Clin. Oncol. 12, 2013-2021.

Cairncross, G., Swinnen, L., Stiff, P., Rosenfeld, S., Vaughan, W., Paleologos, N., Kaminer, L., DeAngelis, L., Nimer, S., Dropcho, E., Peterson, K., Forsyth, P., Stewart, D., Weaver, S., Lieberman, F., Macdonald, D., Ramsay, D., Smith, A. (1997) High-dose thiotepa with hematopoietic reconstitution (deferring radiation) for newly diagnosed aggressive oligodendroglioma. ASCO 16, 388a. (Abstract)

Cairncross, J.G., Ueki, K., Zlatesou, M.C., Lisle, D.K., Finkelstein, D.M., Hammond, R.R., Silver, J.S., Stark, P.C., Macdonald, D.R., Ino, Y., Ramsay, D.A., and Louis, D.N. (1998) Specific genetic predictors of chemotherapeutic response and survival in patients with anaplastic oligodendrogliomas. J. Natl. Cancer Inst. 90, 1473-1479.

Celli, P., Nofrone, I., Palma, L., Cantore, G., and Fortuna, A. (1994) Cerebral oligodendroglioma: Prognostic factors and life history. Neurosurgery 35 , 1018-1035.

Chen, R., Macdonald, D.R., and Ramsay, D.A. (1995) Primary diffuse leptomeningeal oligodendroglioma. Case report. J. Neurosurgery 83, 724-728. 
Dolinskas, C.A., and Simeone, F.A. (1987) CT characteristics of intraventricular oligodendrogliomas. Am. J. Neuroradiol. 8, 1077-1082.

Fortuna, A., Celli, P., and Palma, L. (1980) Oligodendroglioma of the spinal cord. Acta. Neurochir. 52, 305-329.

Frosina, G., Rossi, G., Arena, G., Gentile, S.L., Bruzzone, E., and Abbondandolo, A. (1990) $0^{6}$-alkylguanine-DNA alkyltransferase activity in human brain tumors. Cancer Lett. 55, 153-158.

Gannett, D.E., Wisbeck, W.M., Silbergeld, D.L., and Berger, M.S. (1994) The role of postoperative irradiation in the treatment of oligodendroglioma. Int. J. Radiat. Oncol. Biol. Physics 30, 567-573.

Garza-Mercado, R., Campa, H., and Grajeda, J. (1987) Primary oligodendroglioma of the septum pellucidum. Neurosurgery 21, 78-80.

Glass, J., Hochberg, F.H., Gruber, M.L., Louis, D.N., Smith, D., and Rattner, B. (1992) The treatment of oligodendrogliomas and mixed oligodendroglioma-astrocytomas with PCV chemotherapy. J. Neurosurg. 76, 741-745.

Kallio, M. (1990) Therapy and survival of adult patients with intracranial glioma in a defined population. Acta. Neurol. Scand. 81, 541-549.

Kedar, A., Maria, B.L., Graham-Pole, J., Ringdahl, D.M., QuisIng, R.G., Mickle, J.P., Mendenhall, N.P., Marcus, R.B., Jr., and Gross, S. (1994) High-dose chemotherapy with marrow reinfusion and hyperfractionated irradiation for children with high-risk brain tumors. Med. Pediatr. Oncol. 23, 428-436.

Kros, J.M., Lie, S-T., and Stefanko, S.Z. (1994a) Familial occurrence of polymorphous oligodendroglioma. Neurosurgery 34, 732-736.

Kros, J.M., Pieterman, H., Van Eden, C.G., and Avezaat, C.J. (1994b) Oligodendroglioma: The Rotterdam-Dijkzigt experience. Neurosurgery $\mathbf{3 4}$, 959-966.

Kyritsis, A.P., Yung, W.K.A., Bruner, J., Gleason, M.J., and Levin, V.A. (1993) The treatment of anaplastic oligodendrogliomas and mixed gliomas. Neurosurgery 32, 365-371.

Lee, S.K., and Kelly, D.L., Jr. (1990) Primary oligodendroglioma of the lateral ventricle. South. Med. J. 83, 254-255.

Lee, Y-Y., and Tassel, P. (1989) Intracranial oligodendrogliomas: Imaging findings in 35 untreated cases. Am. J. Roentgenol. 152, 361-369.

Leighton, C., Fisher, B., Bauman, G., Depiero, S., Stitt, L., MacDonald, D., and Cairncross, G. (1997) Supratentorial low-grade glioma in adults: An analysis of prognostic factors and timing of radiation. J. Clin. Oncol. 15, 1294-1301.

Levin, V.A., Edwards, M.S., Wright, D.C., Seager, M.L., Schimberg, T.P., Townsend, J.J., and Wilson, C.B. (1980) Modified procarbazine, CCNU, and vincristine (PCV 3) combination chemotherapy in the treatment of malignant brain tumors. Cancer Treat. Rep. 64, 237-244.

Lindegaard, K-F., Mørk, S.J., Eide, G.E., Halvorsen, T.B., Hatlevoll, R., Solgaard, T., Dahl, O., and Ganz, J. (1987) Statistical analysis of clinicopathological features, radiotherapy, and survival in 170 cases of oligodendroglioma. J. Neurosurg. 67, 224-230.

Ludwig, C.L., Smith, M.T., Godfrey, A.D., and Armbrustmacher, V.W. (1986) A clinicopathological study of 323 patients with oligodendrogliomas. Ann. Neurol. 19, 15-21.

Macdonald, D.R., O'Brien, R.A., Gilbert, J.J., and Cairncross, J.G. (1989) Metastatic anaplastic oligodendroglioma. Neurology 39, 1593-1596.

Macdonald, D.R., Gaspar, L.E., and Cairncross, J.G. (1990) Successful chemotherapy for newly diagnosed aggressive oligodendroglioma. Ann. Neurol. 27, 573-574.

Maintz, D., Fiedler, K., Koopmann, J., Rollbrocker, B., Nechev, S., Lenartz, D., Stangl, A.P., Louis, D.N., Schramm, J., Wiestler, O.D., and von Deimling, A. (1997) Molecular genetic evidence for subtypes of oligoastrocytomas. J. Neuropathol. Exp. Neurol. 56, 1098-1104.

Margain, D., Peretti-Viton, P., Perez-Castillo, A.M., Martini, P., and Salamon, G. (1991) Oligodendrogliomas. J. Neuroradiol. 18, 153-160.

Mason, W.P., Krol, G.S., and DeAngelis, L.M. (1996) Low-grade oligodendroglioma responds to chemotherapy. Neurology 46, 203-207.
Mørk, S.J., Lindegaard, K.-F., Halvorsen, T.B., Lehmann, E.H., Solgaard, T., Hatlevoll, R., Harvei, S., and Ganz, J. (1985) Oligodendroglioma: Incidence and biological behavior in a defined population. J. Neurosurg. 63, 881-889.

Nijjar, T.S., Simpson, W.J., Gadalla, T., and McCartney, M. (1993) Oligodendroglioma: The Princess Margaret Hospital experience (1958-1984). Cancer 71, 4002-4006.

Nutt, C.L., Costello, J.F., Bambrick, L.L., Yarosh, D.B., Swinnen, L.J., Chambers, A.F., and Cairncross, J.G. (1995) $\mathrm{O}^{6}$-methyguanine-DNA methyltransferase in tumors and cells of the oligodendrocyte lineage. Can. J. Neurol. Sci. 22, 111-115.

Pagni, C.A., Canavero, S., and Gaidolfi, E. (1991) Intramedullary "holocord" oligodendroglioma: Case report. Acta. Neurochir. 113, 96-99.

Paleologos, N.A., Zemrus, T., Allen, C.V., Ragin, A., Walsh, A., Lapin, G.D., Groothuis, D.R., and Vick, N.A. (1992) Oligodendrogliomas: CT measurement of transcapillary permeability and response to vincristine chemotherapy. Ann. Neuro. 23, 289. (Abstract)

Paleologos, N., MacDonlad, D., and Vick, N. (1995) Neoadjuvant (pre-radiation $[R T]$ ) procarbazine, $C C N U$ and vincristine (PCV) for anaplastic oligodendroglioma (AO) and aggressive oligodendroglioma (AgO). Neurology 45 (Suppl. 4), A387. (Abstract)

Paleologos, N.A., Vick, N.A., and Kachoris, J.P. (1994) Chemotherapy for lowgrade oligodendrogliomas? Ann. Neurol. 36, 294-295. (Abstract)

Peterson, K., Paleologos, N., Forsyth, P., Macdonald, D.R., and Cairncross, J.G. (1996) Salvage chemotherapy for oligodendroglioma. J. Neurosurg. 85, 597-601.

Reedy, D.P., Bay, J.W., and Hahn, J.F. (1983) Role of radiation therapy in the treatment of cerebral oligodendroglioma:An analysis of 57 cases and a literature review. Neurosurgery 13, 499-503.

Reifenberger, J., Reifenberger, G., Liu, L., James, C.D., Wechsler, W., and Collins, V.P.S (1994) Molecular genetic analysis of oligodendroglial tumors shows preferential allelic deletions on $19 q$ and 1p. Am. J. Pathol. 145, $1175-1190$.

Roosen, N., De La Porte, C., Van Vyve, M., Solheid, C., and Selosse, P. (1984) Familial oligodendroglioma: Case report. J. Neurosurg. 60, 848-849.

Roelvink, N.C.A., Kamphorst, W., Lindhout, D., and Ponssen, H. (1986) Concordant cerebral oligodendroglioma in identical twins J. Neurol. Neurosurg. Psychiatry 49, 706-708.

Rogers, L.R., Estes, M.L., Rosenbloom, S.A., and Harrold, L. (1995) Primary leptomeningeal oligodendroglioma: Case report. Neurosurgery 36, 166-169.

Russell, D.S., and Rubinstein, J.J. (1989) Pathology of Tumors of the Nervous System. Fifth edition. Baltimore: Williams and Wilkins.

Saarinen, U.M., Pihko, H., and Makipernaa, A. (1990) High-dose thiotepa with autologous bone marrow rescue in recurrent malignant oligodendroglioma: A case report. J. Neurooncol. 9, 57-61.

Shaw, E.G., Scheithauer, B.W., O'Fallon, J.R., Tazelaar, H.D., and Davis, D.H. (1992) Oligodendrogliomas: The Mayo Clinic experience. J. Neurosurg. 76, 428-434.

Shaw, E.G., Scheithauer, B.W., O'Fallon, J.R., and Davis, D.H. (1994) Mixed oligoastrocytomas: A survival and prognostic factor analysis. Neurosurgery 34, 577-582.

Shimizu, K.T., Tran, L.M., Mark, R.J., and Selch, M.T. (1993) Management of oligodendrogliomas. Radiology 186, 569-572.

Smith, M.T., Ludwig, C.L., Godfrey, A.D., and Armbrustmacher, V.W. (1983) Grading of oligodendrogliomas. Cancer 52, 2107-2114.

Soffietti, R., Chio, A., Mocellini, C., Rudà, R., Vigliani, M.C., Schiffer, D., Sciolla, R., Seliak, D. (1994) Response of oligodendroglial tumors to PCV chemotherapy. Neurology 44 (Suppl. 2), A309-A310. (Abstract)

Soffietti, R., Chio, A., Mocellini, C., Rudà, R., Schiffer, D., Sciolla, R. (1995) Treatment with carboplatinum of oligodendroglial tumors recurrent after PCV chemotherapy. Neurology 45 (Suppl. 4), A261. (Abstract) 
N. A. Paleologos and J. G. Cairncross: Treatment of oligodendroglioma: An update

Sun, Z.M., Genka, S., Shitara, N., Akanuma, A., and Takakura, K. (1988) Factors possibly influencing the prognosis of oligodendroglioma. Neurosurgery 22, 886-891.

Vonofakos, D., Marcu, H., and Hacker, H. (1979) Oligodendrogliomas: CT patterns with emphasis on features indicating malignancy. J. Comp. Assist. Tomogr. 3, 783-788.

Wallner, K.E., Gonzales, M., and Sheline, G.E. (1988) Treatment of oligodendrogliomas with or without postoperative irradiation. J. Neurosurg. 68, 684-688.
Wiestler, O., Kleihues, P., and Pegg, A.E. (1984) O ${ }^{6}$-alkylguanine-DNA alkyltransferase activity in human brain and brain tumors. Carcinogenesis $\mathbf{5}$, 121-124.

Wilkinson, I.M.S., Anderson, J.R., and Holmes, A.E. (1987) Oligodendroglioma: An analysis of 42 cases. J. Neurol. Neurosurg. Psychiatry 50, 304-312.

Yuen, S.T., Fung, C.F., Ng, T.H., and Leung, S.Y. (1992) Central neurocytoma: its differentiation from intraventricular oligodendroglioma. Childs Nerv. Syst. 8, 383-388. 